\title{
Proton pump inhibitor-responsive oesophageal eosinophilia: an entity challenging current diagnostic criteria for eosinophilic oesophagitis
}

\author{
Javier Molina-Infante, ${ }_{1}^{1}$ Albert J Bredenoord, ${ }^{2}$ Edaire Cheng, ${ }^{3}$ Evan S Dellon, ${ }^{4}$ \\ Glenn T Furuta, ${ }^{5}$ Sandeep K Gupta, ${ }^{6}$ Ikuo Hirano, ${ }^{7}$ David A Katzka, ${ }^{8}$ \\ Fouad J Moawad, ${ }^{9}$ Marc E Rothenberg, ${ }^{10}$ Alain Schoepfer, ${ }^{11}$ Stuart I Spechler, ${ }^{12}$ \\ Ting Wen, ${ }^{10}$ Alex Straumann, ${ }^{13}$ Alfredo J Lucendo, ${ }^{14}$ From the PPI-REE Task Force \\ of the European Society of Eosinophilic Oesophagitis (EUREOS)
}

For numbered affiliations see end of article.

\section{Correspondence to Dr Javier Molina-Infante, Department of Gastroenterology, Hospital San Pedro de Alcantara, C/Pablo Naranjo s/n, Caceres 10003, Spain; \\ xavi_molina@hotmail.com}

Received 29 October 2015 Revised 17 November 2015 Accepted 22 November 2015 Published Online First 18 December 2015

\section{ABSTRACT}

Consensus diagnostic recommendations to distinguish GORD from eosinophilic oesophagitis (EoE) by response to a trial of proton pump inhibitors (PPIs) unexpectedly uncovered an entity called 'PPI-responsive oesophageal eosinophilia' (PPI-REE). PPI-REE refers to patients with clinical and histological features of EOE that remit with PPI treatment. Recent and evolving evidence, mostly from adults, shows that patients with PPI-REE and patients with EoE at baseline are clinically, endoscopically and histologically indistinguishable and have a significant overlap in terms of features of Th2 immune-mediated inflammation and gene expression. Furthermore, PPI therapy restores oesophageal mucosal integrity, reduces Th2 inflammation and reverses the abnormal gene expression signature in patients with PPIREE, similar to the effects of topical steroids in patients with EoE. Additionally, recent series have reported that patients with EoE responsive to diet/topical steroids may also achieve remission on PPI therapy. This mounting evidence supports the concept that PPI-REE represents a continuum of the same immunological mechanisms that underlie EoE. Accordingly, it seems counterintuitive to differentiate PPI-REE from EoE based on a differential response to PPI therapy when their phenotypic, molecular, mechanistic and therapeutic features cannot be reliably distinguished. For patients with symptoms and histological features of EoE, it is reasonable to consider PPI therapy not as a diagnostic test, but as a therapeutic agent. Due to its safety profile, ease of administration and high response rates (up to 50\%), PPI can be considered a first-line treatment before diet and topical steroids. The reasons why some patients with EoE respond to PPI, while others do not, remain to be elucidated.

\section{HISTORICAL BACKGROUND AND DEFINITIONS}

Eosinophilic oesophagitis (EoE) and GORD are the most prevalent chronic oesophageal inflammatory conditions in children and adults in the Western world. ${ }^{1}$ Whereas the first is an allergen-driven disease, ${ }^{2}$ the latter develops as a consequence of pathological exposure of the oesophageal mucosa to acid-predominant gastric contents. ${ }^{1}$ Distinguishing both disorders is important because of their different aetiopathogenesis, natural history and monitoring. $^{2}$ However, a rigid distinction between EoE and GORD is difficult due to overlapping clinical and histological features, not to mention their frequent coexistence and potential partially shared pathogenic pathways. ${ }^{3}$ The presence of heartburn and marked oesophageal eosinophilia, for instance, might be fairly common in both entities. ${ }^{3}{ }^{4}$ In paediatric patients, this differentiation is even more complex due to a wider spectrum of clinical manifestations, difficulties in expressing symptoms and subtle or absent endoscopic abnormalities. ${ }^{5}$

In order to solve this diagnostic conundrum, the first consensus recommendations for diagnosis and management of EoE were published in $2007 .{ }^{6}$ These guidelines advocated a diagnosis of EoE in patients with symptomatic oesophageal eosinophilia ( $>15$ eosinophils per high power field (eos/HPF)) showing either lack of response to proton pump inhibitor (PPI) therapy or a normal acid exposure on oesophageal $\mathrm{pH}$ monitoring. Accordingly, a diagnosis of GORD was recommended for those patients who were either responders to PPI therapy or had objective evidence of pathological oesophageal acid exposure. This distinction was based on the assumption that only GORD, as an acid-related disorder, could respond to the acid-suppressive effect of PPIs. As such, these guidelines equated GORD with symptomatic and histological response to PPI therapy. Far from fulfilling the expectation of distinguishing GORD from EoE, the recommended PPI trial unexpectedly uncovered a third intriguing category of patients apparently sharing features of EoE and GORD. ${ }^{4}$

Updated consensus recommendations in $2011^{2}$ included changes to these findings: (1) the description of a novel phenotype, PPI-responsive oesophageal eosinophilia (PPI-REE), referring to patients with features of EoE who achieve clinical and histological remission on PPI therapy (2) response to PPI therapy in patients with PPI-REE was not necessarily considered a manifestation of GORD and (3) the retraction of recommending oesophageal $\mathrm{pH}$ monitoring as a diagnostic criterion, due to its low accuracy to predict response to PPI. ${ }^{4}$ Nonetheless, support for a PPI trial was maintained as a diagnostic criterion, since PPI-REE and EoE were still considered separate clinical entities as they showed a different response to the PPI trial. ${ }^{2}$

At this stage, it is crucial to ascertain the accurate location of PPI-REE within the spectrum between EoE and GORD, the therapeutic mechanisms 
leading to responsiveness to PPI therapy in patients with suspected EoE and whether the response to a PPI trial has any validity as a means of excluding EoE.

\section{DIFFERENCES AND SIMILARITIES BETWEEN GORD, PPI-REE AND EOE}

The need to distinguish among GORD, EoE and PPI-REE in clinical practice, pharmaceutical trials and research studies has led to careful investigations to distinguish these entities. The results of these studies are summarised in table 1.

\section{Symptoms}

In adults, the clinical presentations of GORD and EoE are typically distinct. ${ }^{3}$ Patients with GORD present with heartburn, regurgitation and bitter/sour taste of gastric content. Dysphagia as a dominant symptom is rare in GORD, unless a peptic stricture is present. GORD symptoms are exacerbated after consumption of large meals, rapid eating, acidic foods, alcohol, obesity, tobacco and body position changes. In contrast, adult patients with EoE present predominantly with intermittent dysphagia during consumption of solid foods commonly associated with food impactions. While heartburn and chest pain may be present in EoE, they are characteristically not the dominant complaints reported by adult patients and if present, usually accompany dysphagia. Available studies have identified that demographics, atopic history and clinical manifestations do not reliably discriminate EoE from PPI-REE. ${ }^{4-11}$ Paediatric presentations of EoE are more heterogeneous and include abdominal pain, nausea, reflux-like symptoms not responsive to acid suppression, feeding difficulties and growth failure. It remains unclear if this difference in symptom profile reflects inadequate symptom reporting by young children, initial symptoms related to inflammation prior to onset of oesophageal remodelling or in part functional symptoms caused by comorbid conditions, such as irritable bowel syndrome. Along the same lines, it is unclear if adults with EoE only develop dysphagia after an initial period of paediatric type symptoms. ${ }^{5}$

\section{Endoscopic features}

Most patients with GORD have a normal appearance of the oesophageal mucosa on endoscopy, whereas erosive oesophagitis or Barrett's oesophagus is identified in the minority. ${ }^{3}$ Endoscopically, nearly all adult patients with EoE demonstrate one or more characteristic features of loss of vascular markings, rings, white exudates, longitudinal furrows, narrow calibre oesophagus and strictures, whereas some children may have a visually normal mucosa. ${ }^{11}{ }^{12}$ Reflecting the natural history of oesophageal remodelling, rings and strictures are common in adults but rare findings in children with EoE. ${ }^{5}$ Typical EoE endoscopic signs are useful in distinguishing GORD from EoE, but not PPI-REE from EoE. ${ }^{4} 81011$

\section{Histological findings}

Histological characteristics of GORD include basal cell hyperplasia, papillary elongation, dilated intracellular spaces and a paucity of intraepithelial inflammatory cells. ${ }^{13}$ Eosinophils may be present in GORD but typically are in low numbers $(<10$ eos/ HPF), although we lack prospective studies defining numbers and extent and numbers of eosinophils observed in GORD. Histological features of EoE include all of the above GORD features with the addition of a marked, eosinophil-predominant, cellular infiltration of the mucosa. Superficial squamous epithelial distribution, eosinophil degranulation, eosinophil microabscesses and lamina propria fibrosis are also commonly identified in EoE, but not in GORD. Mast cells have been recognised in the mucosa of both patients with GORD and patients with EoE. ${ }^{14}{ }^{15}$ Multiple studies have noted that these histological features are found in both EoE and PPI-REE. These include evidence of superficial distribution of epithelial eosinophils, eosinophil degranulation and microabscess formation, ${ }^{4}{ }^{7} 811$

Table 1 Updated similarities and differences between GORD, PPI-REE and EoE

\begin{tabular}{|c|c|c|c|}
\hline & GORD & PPI-REE & EoE \\
\hline Age & Adults >children & Children and young adults & Children and young adults \\
\hline Gender & Male=Female & Male predominance & Male predominance \\
\hline Dominant symptom & Heartburn, regurgitation & Dysphagia & Dysphagia \\
\hline Food impaction & Uncommon & Common & Common \\
\hline Endoscopic findings & $\begin{array}{l}\text { Normal endoscopy }(70-80 \%) \\
\text { Erosions, ulcers, strictures, } \\
\text { Barrett's oesophagus, } \\
\text { oesophageal adenocarcinoma }\end{array}$ & $\begin{array}{l}\text { Normal endoscopy }(<10 \%) \\
\text { Oedema, rings, exudates furrows, strictures, } \\
\text { crêpe-paper oesophagus, narrow calibre oesophagus }\end{array}$ & $\begin{array}{l}\text { Normal endoscopy }(<10 \%) \\
\text { Oedema, rings, exudates. } \\
\text { furrows, strictures, crêpe-paper oesophagus, } \\
\text { narrow calibre oesophagus }\end{array}$ \\
\hline $\begin{array}{l}\text { Histology and inflammatory } \\
\text { cells }\end{array}$ & $\begin{array}{l}\text { Usually }<5-10 \text { eos/HPF } \\
\text { Neutrophils, lymphocytes, } \\
\text { low-grade eosinophilia }\end{array}$ & $\begin{array}{l}>15 \text { eos/HPF } \\
\text { Eosinophils and mast cells }\end{array}$ & $\begin{array}{l}>15 \text { eos/HPF } \\
\text { Eosinophils and mast cells }\end{array}$ \\
\hline $\begin{array}{l}\text { Oesophageal acid exposure on } \\
\text { pH monitoring }\end{array}$ & Mostly positive & Positive and negative & Negative and positive \\
\hline Primary treatment & $\begin{array}{l}\text { Inhibitors of gastric acid } \\
\text { secretion, including } \\
\text { PPIs, surgical fundoplication }\end{array}$ & $\begin{array}{l}\text { PPI therapy, unclear whether other inhibitors of } \\
\text { gastric acid secretion are effective }\end{array}$ & $\begin{array}{l}\text { Topical steroids } \\
\text { Elimination diet }\end{array}$ \\
\hline Aetiology & Reflux of gastric contents & Unclear & Food/airborne allergens \\
\hline $\begin{array}{l}\text { Type of immune response/ } \\
\text { involved chemo/cytokines }\end{array}$ & $\begin{array}{l}\text { Th1 } \\
\text { IL-8, MCP-1, RANTES }\end{array}$ & $\begin{array}{l}\text { Th2 } \\
\text { Eotaxin-3, IL-5, IL-13 }\end{array}$ & $\begin{array}{l}\text { Th2 } \\
\text { Eotaxin-3, IL-5, IL-13 }\end{array}$ \\
\hline EoE transcriptome panel & Not expressed & Similar expression to EoE & Similar expression to PPI-REE \\
\hline $\begin{array}{l}\text { Specific molecular effect of } \\
\text { therapy }\end{array}$ & - & $\begin{array}{l}\text { PPIs downregulate Th2 inflammation and normalise } \\
\text { EoE gene expression }\end{array}$ & $\begin{array}{l}\text { Topical steroids downregulate Th2 } \\
\text { inflammation and normalise EoE gene } \\
\text { expression }\end{array}$ \\
\hline
\end{tabular}

EoE, eosinophilic oesophagitis; IL, interleukin; MCP-1, monocyte chemoattractant protein-1; PPI, proton pump inhibitor; REE, responsive oesophageal eosinophilia. 
basophil infiltration ${ }^{10}$ and the expression of major basic protein and tryptase. ${ }^{15}$ Interestingly, a lower rate of response to PPI therapy has been reported in patients with more severe histological findings, including either $\geq 15$ eos/HPF at three levels of biopsies $^{16}$ or increasing degrees of oesophageal eosinophilia. ${ }^{4}$

\section{Molecular and genetic features}

GORD promotes a proinflammatory response characterised by innate immunity with overexpression of cytokines, such as interleukin (IL)-8 (CXCL8), CCL2 (monocyte chemoattractant protein-1) and CCL5 (Regulated on Activation, Normal T Expressed and Secreted (RANTES)). ${ }^{17}$ These cytokines and chemokines promote active recruitment of neutrophils and lymphocytes and sometimes a mild eosinophilic infiltration, normally $<5-10$ eos/HPF. Unlike GORD, EoE is a chronic immunoallergic disorder characterised by an aberrant Th2 inflammatory response involving IL-5 and IL-13 and local production of CCL26 (eotaxin-3), a chemokine that specifically attracts eosinophils to the oesophageal mucosa. When activated, the eosinophils cause local tissue damage and recruit and/or activate other effector cells, such as mast cells, which have a role in oesophageal fibrotic remodelling. ${ }^{18}$ By using whole-genome transcript expression profiling of oesophageal tissue, a molecular EoE diagnostic panel has been recently identified. ${ }^{19}$ This panel is made of 94 EoE genes and accurately distinguishes patients with EoE from GORD or control subjects. ${ }^{19}$

Over the past years, an increasing number of papers have tried to further characterise PPI-REE. Baseline markers of eosinophilic inflammation in oesophageal tissue (eg, eosinophil-derived major basic protein and CCL26) have been shown to be increased in PPI-REE similar to EoE. In addition, the expression of mast cell signature genes (eg, tryptase), ${ }^{15}$ as well as the expression of genes involved in type 2 (Th2)-associated allergic inflammation (including CCL26, IL-5, IL-13, thymic stromal lymphopoietin (TSLP) and periostin (POSTN)) 9102021 have demonstrated largely overlapping patterns between EoE and PPI-REE, although PPI-REE typically has more modest overexpression levels. One of the key findings in the past year is that PPI-REE, unlike GORD, has a transcriptome that nearly completely overlaps with the EoE transcriptome, including the hallmark EoE genes for eosinophil chemotaxis (CCL26), barrier molecules (desmoglein DSG1), tissue remodelling (POSTN) and mast cells $(C P A 3) .{ }^{22}$ Overall, these findings suggest PPI-REE and EoE are alike and both associated with allergic inflammation (4). In addition, recent clinical studies have shown that PPI monotherapy in patients with PPI-REE can almost completely reverse the Th2 signature of PPI-REE (CCL26, IL-5, IL-13, POSTN $)^{9} 2122$ and concurrently induce a normalisation of the mast cell genes (CPA3, TPSAB2), Th2 inflammation indicators (TNFAIP6, ALOX15), epithelial barrier genes (DSG1, CDH26, FLG), tissue fibrosis markers (eg, KRT13) and IL-13/ IL-4-induced genes (POSTN, MUC4). ${ }^{22}$ Since these effects are similar to those of topical steroids in patients with EoE, ${ }^{9} 23$ these striking data pose the possibility that EoE and PPI-REE represent a common disorder.

Recent genome-wide association studies in EoE have identified two replicated susceptibility loci at $2 \mathrm{p} 23$ and $5 \mathrm{q} 22$, regions that encode the epithelial gene products CAPN14 and TSLP. ${ }^{24-26}$ The presence of susceptibility loci was shown to not depend upon response to PPI, reinforcing the idea that oesophageal eosinophilia, independent of PPI stratification, likely shares genetic aetiology.

\section{THE EFFICACY OF PPI THERAPY IN PATIENTS WITH SUSPECTED EOE}

The evidence for PPIs inducing either clinical or histological disease remission in patients with suspected EoE was initially reported by one case series and three retrospective studies published between 2005 and 2009. ${ }^{16} 27-29$ The case series reported clinicohistological response in all three patients, ${ }^{27}$ whereas a $50-86 \%$ clinical response and a $40 \%$ histological response were reported in the retrospective cohorts. ${ }^{16} 28$ In the first large prospective study in adults with clinical, endoscopic and histological features of EoE, an 8 -week course of PPI therapy led to complete response in $50 \%(5 / 10)$ of cases. ${ }^{4}$ Of note, response to PPIs was observed not only in $80 \%(23 / 29)$ of patients with endoscopic evidence of GORD or abnormal $\mathrm{pH}$ monitoring, but also in $33 \%$ of those with a normal $\mathrm{pH}$ study. ${ }^{4}$

Two randomised controlled trials comparing PPIs with topical steroids in patients with an EoE phenotype reported a similar efficacy $(33 \%)$ for PPI therapy. ${ }^{30} 31$ The latter trial demonstrated a response to PPIs in $100 \%(4 / 4)$ and $18 \%(3 / 17)$ of patients with a pathological $\mathrm{pH}$ study and normal $\mathrm{pH}$ study, respectively. ${ }^{31}$ In three recent prospective studies, $35-47 \%$ of adult and paediatric patients achieved histological remission (defined by $<5$ eos/HPF) on PPI therapy. ${ }^{15} 3233$ Of note, response to PPI therapy increased up to $50 \%,{ }^{30} 57 \%{ }^{32}$ and $68 \% 0^{33}$ when histological remission was redefined as $<15$ eos/ HPF.

A recent systematic review with meta-analysis, including 33 studies with 619 patients with suspected EoE, revealed that PPIs achieved histological remission (defined by $<15$ eos/HPF) in $51 \%(95 \%$ CI $42.2 \%$ to $58.7 \%)$ and symptomatic improvement in $61 \%$ (95\% CI $48.38 \%$ to $72.2 \%$ ) of cases. ${ }^{34}$ No significant differences were noted in patients' age, study design and types of PPIs assessed. However, a trend towards increased efficacy was observed when PPIs were administered twice daily compared with once daily, and among patients with increased oesophageal acid exposure on $\mathrm{pH}$ monitoring. Noteworthy, a significant publication bias in favour of studies reporting histological responses to PPI therapy was observed in this meta-analysis.

The sustained efficacy of PPIs in children has been evaluated in two retrospective small series and a recent prospective study, with most patients $(11 / 14,78.6 \%)$ remaining in clinicopathological remission at 1-year follow up while on maintenance PPI therapy. ${ }^{33} 3536$ As for adults, the first long-term follow-up multicentre study including 75 patients with PPI-REE demonstrated that the majority of patients $(55 / 75,73 \%)$ maintained histological remission 1 year after tapering dosage to the lowest effective clinical dose. ${ }^{37}$ Among relapsers, most regained histological remission with dose escalation, suggesting some patients with PPI-REE continue to require high-dose maintenance PPI. Allergic rhinoconjunctivitis and a CYP2C19 rapid metabolizer genotype predicted long-term relapse, hinting at the influence of pharmacogenomic and environmental factors on the longterm efficacy of PPI therapy.

\section{POTENTIAL MODE OF ACTION OF PPIS IN EOE}

It is widely appreciated that PPIs block gastric acid secretion, and this antisecretory effect is assumed to underlie their great efficacy in treating GORD. However, PPIs do not prevent the reflux of non-acidic material, and up to $20 \%$ of patients with GORD have symptoms that are refractory to PPIs. It is less well known that PPIs have anti-inflammatory actions (independent of antisecretory effects) that also might contribute to healing 
oesophagitis. $^{38}$ PPIs have antioxidant properties, inhibit immune cell functions, decrease adhesion molecule expression by endothelial cells and reduce inflammatory cytokine expression by epithelial cells. ${ }^{39}$ PPIs also have anti-inflammatory effects that might be especially pertinent to the allergen-driven eosinophilia of EoE.

In EoE, eosinophils accumulate in the oesophagus when allergens induce production of Th2 cytokines like IL-4 and IL-13, which stimulate oesophageal secretion of CCL26 (eotaxin-3). ${ }^{18}$ Omeprazole, in concentrations achieved in blood with conventional dosing, inhibits Th2 cytokine-stimulated eotaxin-3 secretion in isolated oesophageal epithelial cells by blocking binding of the transcription factor STAT6 to the eotaxin-3 promoter. ${ }^{40} 41$ Lansoprazole exhibits similar actions, suggesting that this inhibition of Th2 cytokine-stimulated eotaxin-3 secretion is a PPI drug class effect. ${ }^{41}$ In one study of children with oesophageal eosinophilia, PPI treatment significantly decreased eotaxin-3 protein expression by epithelial cells in the proximal but not distal oesophagus. ${ }^{42}$ In three recent studies primarily in adult patients with PPI-REE, ${ }^{9} 2122$ PPIs reduced oesophageal expression of eotaxin-3, IL-5 and mast cell density, suggesting that PPIs downregulate Th2-mediated events. Moreover, gene transcriptome analyses of oesophageal biopsies from adult and paediatric patients with PPI-REE have shown a pronounced and specific effect of PPIs on reducing expression of genes related to allergic inflammation. ${ }^{22}$ Impaired oesophageal mucosal barrier function, likely mediated by reduced expression of desmoglein-1, is a common feature of EoE, and PPIs have been shown to restore mucosal barrier function and improve desmoglein-1 expression in patients with PPI-REE. ${ }^{21} 2243$

All of the therapeutic effects of PPIs on oesophageal inflammation, gene expression and mucosal integrity in patients with PPI-REE are similar to the responses seen with topical steroid therapy in patients with EoE. ${ }^{94}$ Collectively, these data support a trial of PPIs for virtually any patient with oesophageal eosinophilia, regardless of the underlying mechanism. ${ }^{45}$ If eosinophilia is caused solely by GORD and is not antigen driven, then PPI antisecretory effects can improve eosinophilia by limiting acid reflux. If oesophageal eosinophilia is solely antigen driven, antiinflammatory PPI effects might improve eosinophilia by attenuating Th2-associated responses. If GORD causes or exacerbates an antigen-driven oesophageal eosinophilia, both the antisecretory and anti-inflammatory effects of PPIs might combine to ameliorate the condition. Finally, hypersensitivity to acid in the oesophagus has been reported in patients with EoE. ${ }^{46}$ During perfusion of the oesophagus with acid, patients with EoE felt the burning sensation evoked by the acid earlier than those with concomitant reflux or healthy volunteers. ${ }^{46}$ This phenomenon might explain why PPI-mediated acid suppression may improve symptoms in some patients with EoE, despite the absence of histological remission on PPI therapy. $49162847-49$

\section{PPI-REE: IS IT GORD OR IS IT EOE?}

The above-mentioned data all point in the same direction suggesting that PPI-REE and EoE are indistinguishable except that PPIs have a more robust effect on patients with PPI-REE than patients with EoE. Subjects with EoE and PPI-REE have similar symptoms, demographics, endoscopic findings, histology and response to other treatments besides PPIs. Most striking, perhaps, is that the transcriptomes of EoE and PPI-REE largely overlap. Furthermore, recent data reveal that patients with EoE responsive to diet and topical steroid therapy were eventually found to respond to PPI therapy as well, providing further data that an allergic inflammatory cause is important in PPI-REE. 5051

All of these data provide no rational basis to make a distinction between patients with symptomatic oesophageal eosinophilia based on a different response to PPI therapy. At the present time, phenotypic, molecular, mechanistic and therapeutic features cannot reliably distinguish EoE from PPI-REE. As such, the requirement of a distinct name among indistinguishable patients for the subgroup responding to PPIs is questionable. ${ }^{52}$ We therefore propose not to include the responsiveness to a given drug as a diagnostic criterion and, consequently, avoiding the term PPI-REE for those subjects who have an EoE phenotype with both histological and clinical responses to PPI therapy. Given all of the above-mentioned arguments, we suggest viewing the PPI trial not as a diagnostic tool for EoE, but rather as a potential therapy in all patients with clinical, endoscopical and histological features suitable for EoE.

\section{REAPPRAISAL OF THE PPI TRIAL AS A DIAGNOSTIC TOOL AND POSITION OF PPIS IN THE TREATMENT OF EOE}

Currently, either swallowed topical steroids or dietary elimination are considered an appropriate first-line therapeutic options after the diagnosis of EoE is established, ${ }^{53}$ but these modalities have limitations and neither is universally effective. ${ }^{55} 56$ Therefore, it is important to consider where PPIs might fit in the treatment algorithm for EoE. Respecting their favourable safety profile, the simplicity of administration of the compounds and high response rates, ${ }^{34}$ PPIs could be considered as first-line therapy for patients with EoE. The use of PPIs would therefore, instead of deciphering which patients do not have EoE, will likely identify a substantial proportion of patients with EoE who achieve remission on PPI therapy and will not need topical steroid or dietary therapy. As with topical steroid use, it is important to note that this represents off-label use of these medications.

\section{PROPOSAL FOR UPDATED DIAGNOSTIC CRITERIA FOR EOE}

EoE represents a chronic, immune/antigen-mediated oesophageal disease characterised clinically by symptoms related to oesophageal dysfunction and histologically by eosinophilpredominant inflammation. Eosinophilic inflammation is restricted to the oesophagus and other causes of local and systemic oesophageal eosinophilia should be excluded (box 1).

After a diagnosis of EoE, clinical and histological features of EoE may respond in the majority of patients to treatment with PPIs, topical steroids or elimination diets.

\section{UNSOLVED ISSUES}

\section{Can we positively state that PPI-REE is EoE?}

No, we cannot. EoE is formally defined as an immune/antigenmediated disease, but we currently lack evidence on the ultimate aetiology of PPI-REE. Solid evidence corroborates it is a Th2-mediated disease with significant molecular overlap with EoE, but we do not know whether this immune response is triggered by reflux-mediated epithelial injury, food/airborne allergens or the combination of both factors.

In addition, a diagnosis of EoE in patients with no clinical or endoscopic features of EoE might be questionable, given the fact we know patients with GORD might also have Th1-mediated oesophageal eosinophilia. ${ }^{22}$ However, this subset of patients is likely to represent a minority of adult patients. A recent study performed a thorough subanalysis of 75 patients with PPI-REE on long-term follow-up and $86 \%(64 / 75)$ of patients had typical clinical and endoscopic features of EoE, 
Box 1 Proposal for updated diagnostic criteria for eosinophilic oesophagitis (EoE)

1. Symptoms of oesophageal dysfunction (dysphagia/food impaction in adults; abdominal pain, nausea, reflux-like symptoms, feeding difficulties, growth failure, dysphagia in children)

2. Baseline oesophageal eosinophil-predominant inflammation (characteristically consisting of a peak value of $\geq 15$ eos/HPF) limited to the oesophagus

- Baseline endoscopy should be preferably performed off proton pump inhibitor (PPI) therapy to better understand the patient profile in case of further response to PPI therapy

- Other local and systemic causes of oesophageal eosinophilia should be ruled out: eosinophilic gastroenteritis, Crohn's disease, hypereosinophilic syndrome, parasites, drug hypersensitivity, achalasia, vasculitis, pemphigoid, connective tissue disorders and graft-versus-host disease

- Biopsies from the antrum and/or duodenum should be obtained in all children and in adults with Gl symptoms or endoscopic abnormalities

- A diagnosis of EoE in patients based solely on histology, without clinical and endoscopic features compatible with EoE, might be questionable

- Routine oesophageal pH monitoring is not recommended in the diagnostic work-up of EoE

- A majority of patients with EoE will achieve symptom response and histological remission ( $<15$ eos/HPF) on PPI, topical steroid or dietary intervention

with only one single patient showing a pure GORD phenotype. $^{37}$ The bulk of evidence on PPI-REE comes from adult patients, so we need further prospective studies corroborating these findings in children as well. Based on the high population prevalence of GORD, it is inevitable that many patients with EoE will have coexisting GORD. In such cases or atypical clinical presentations, comprehensive consideration of the clinical criteria listed in table 1, endoscopic features, ambulatory $\mathrm{pH}$ monitoring and responsiveness to PPI therapy may have clinical utility in patient management.

Molecular biomarkers distinguishing EoE and PPI-REE would be helpful to distinguish between both entities. KCNJ2 has been recently identified as the only gene with significant differential expression between PPI-REE and EoE, showing a 72\% sensitivity/specificity to predict PPI-REE at baseline. ${ }^{22}$ KCNJ2 encodes a potassium channel which is abundant in GI mucosa and colocalises with the proton pump. Therefore, the authors proposed a potential interaction between this potassium channel and proton pump in the upper GI epithelium to explain PPI-REE. A genome-wide approach currently underway may reveal alternative mechanisms that might differentiate the two entities.

\section{Considerations for paediatric patients}

A distinction between EoE and GORD may be especially complex in children, where EoE symptoms tend to overlap more substantially with GORD (feeding difficulties, regurgitation, heart burn) and endoscopic findings are not so prototypical as in adults. Concerns about endoscopic procedures in children often lead to treatment with PPIs before any diagnostic procedures are completed. A symptomatic response to PPIs will lead to most paediatricians considering a diagnosis of GORD, but a diagnosis of PPI-REE might be missed since biopsies were not obtained. Furthermore, a significant dissociation between oesophageal symptoms and inflammation has been reported in EoE, so a clinical response to PPI therapy does not necessarily rule out EoE. ${ }^{4} 162847-49$ Unfortunately, EoE is a clinicohistological entity requiring objective confirmation of histological abnormalities for diagnosis and for remission after therapeutic interventions.

Performing an additional baseline endoscopy off PPI therapy raises concerns for practitioners, parents and patients, but it is critical to remember that normal endoscopic and histological oesophageal features on PPI therapy in children with suspected EoE could create a lack of diagnostic clarity as well as shortterm and long-term therapeutic uncertainties. For instance, children with GORD, PPI-REE, functional dyspepsia or recurrent abdominal pain might have similar symptoms (regurgitation, vomiting, abdominal pain), experience a therapeutic-related or a placebo-related response to PPIs and exhibit normal endoscopic and histological features on PPI therapy. Questions of the duration, dose and frequency of PPI treatment will remain unanswered. Overall, reconciling concerns about endoscopic procedures and anaesthesia with the current need of endoscopy for diagnosis and monitoring EoE will continue to be challenging in paediatric patients.

\section{Mechanisms underlying response to PPI therapy}

The precise mechanism(s) by which PPIs accomplish their effects on oesophageal eosinophilia in EoE remains unclear. Anti-inflammatory effects of PPIs have been only proven in experimental studies. ${ }^{40}$ While omeprazole in vitro is present in the culture media for up to $48 \mathrm{~h}$, the short half-life for PPI drugs (1-2 h active) makes it unclear if a sustained antiinflammatory effect is maintained in vivo. PPI therapy have recently shown their ability to downregulate Th2 allergic oesophageal inflammation, ${ }^{921} 22$ but it is not certain whether this is a direct (primary anti-inflammatory effect) or indirect (primary acid inhibition leads to secondary inflammation healing) effect.

On the other hand, the role of GORD in PPI-REE is unclear. PPIs can reverse dilation of epithelial intercellular spaces and restore mucosal integrity in patients with $\mathrm{GORD}^{57}$ and PPI-REE ${ }^{21} 43$ suggesting reflux may be the initial trigger in some patients with PPI-REE. This hypothesis might be supported by a greater likelihood of GORD in patients with PPI-REE. ${ }^{34}$ However, the demonstration of pathological oesophageal acid exposure in patients with PPI-REE does not prove a casual role for GORD, whereas lack of response to PPIs does not necessarily rule out GORD as a primary trigger for EoE. It will be important to eventually determine if patients with PPI-REE would also respond to other classes of anti-acid drugs such as histamine receptor 2 (H2R) antagonists, as it would be informative of the acid-suppressive effects as a primary driver of the PPI-REE designation. It is important to acknowledge that no complete response of another allergic disease with PPI therapy has been documented so far today.

\section{How do we define response to PPI therapy?}

The effect of PPIs in patients with suspected EoE is not an all or none effect, but a gradient varying between no response, some response and near-complete or complete response. It should be emphasised that, currently, a diagnosis of EoE which 
Table 2 PPI doses and duration and response rates in prospective studies evaluating PPI-REE

\begin{tabular}{|c|c|c|c|c|}
\hline First author, year of publication & Drug, doses & Dosing interval & Duration (weeks) & $\begin{array}{l}\text { Histological remission } \\
\text { rates after PPI therapy (definition) }\end{array}$ \\
\hline Peterson, $2010^{30}$ & Esomeprazole $40 \mathrm{mg}$ & Once daily & 8 & $\begin{array}{l}33 \%(<5 \text { eos/HPF }) \\
50 \%(<15 \text { eos/HPF })\end{array}$ \\
\hline Molina-Infante, $2011^{4}$ & Rabeprazole $20 \mathrm{mg}$ & Twice daily & 8 & $50 \%(<15$ eos/HPF $)$ \\
\hline Francis DL, $2012^{47}$ & Esomeprazole $40 \mathrm{mg}$ & Twice daily & 6 & $61 \%$ (average $<5$ eos/HPF) \\
\hline Moawad, $2013^{31}$ & Esomeprazole $40 \mathrm{mg}$ & Once daily & 8 & $33 \%(<7$ eos/HPF $)$ \\
\hline Dellon, $2013^{7}$ & Any of the PPI drugs at $20-40 \mathrm{mg}$ & Twice daily & 8 & $36 \%(<15$ eos/HPF) \\
\hline Vazquez-Elizondo, $2013^{32}$ & Omeprazole $20 \mathrm{mg}$ & Twice daily & 8 & $\begin{array}{l}25 \%(<5 \text { eos/HPF }) \\
56 \%(<15 \text { eos/HPF })\end{array}$ \\
\hline Molina-Infante, $2014^{9}$ & Omeprazole $40 \mathrm{mg}$ & Twice daily & 8 & $43 \%(<15$ eos/HPF $)$ \\
\hline Van Rhijn, $2014^{21}$ & Esomeprazole $40 \mathrm{mg}$ & Twice daily & 8 & $50 \%(<15$ eos/HPF $)$ \\
\hline Gutiérrez-Junquera, $2015^{33}$ & Esomeprazole $1 \mathrm{mg} / \mathrm{kg} /$ dose & Twice daily & 8 & $\begin{array}{l}47 \%(<5 \text { eos/HPF }) \\
68.6 \%(<15 \text { eos/HPF })\end{array}$ \\
\hline
\end{tabular}

responds to PPI therapy depends on subjective criteria for symptom response and on an arbitrary histological cut-off (15 eos/HPF) for histological response. It is likely that PPI use will have at least some effect in most patients, suggesting that either the acid inhibitory and/or the anti-inflammatory effect of PPIs may play a smaller or larger role in these patients.

\section{Adequate doses, dosing interval and duration of PPI therapy}

It is also necessary to determine the dose and duration of an adequate initial PPI trial. An 8-week course of any of the available agents at a regular dose twice daily (pantoprazole $40 \mathrm{mg}$, rabeprazole $20 \mathrm{mg}$, lansoprazole $30 \mathrm{mg}$; all twice daily) or double dose once daily (omeprazole $40 \mathrm{mg}$, esomeprazole $40 \mathrm{mg}$ ) has been proposed as sufficient to assess a response to PPI therapy. ${ }^{2}$ In young children, dosing should be weight based as appropriate. However, evidence supporting the recommendations is poor and conflicting. ${ }^{34}$ While there does not seem to be a relation between the medication dose and response rate in prospective studies, it is clear that any of the PPI agents can be effective when used at a 'high daily dose' (table 2). The first meta-analysis on this issue has recently suggested a nonstatistically significant advantage of a twice daily administration, with no differences between drugs or doses. ${ }^{34}$ Future prospective dose-ranging studies of PPIs in patients with oesophageal eosinophilia would be helpful in providing more definitive dose and duration recommendations.

\section{Natural history and long-term prognosis of responders to PPI therapy}

The similarities between PPI-REE and EoE also raise the question of whether oesophageal fibrotic remodeling is present if left unmanaged, or whether PPI therapy can lead to reversal of oesophageal fibrosis in PPI-REE. ${ }^{58}$ Further studies should address this issue.

\section{Combination therapy: PPIs plus steroid/diet therapy}

Another area of speculated use is in combined therapy with steroids, particularly for refractory patients. This would combine PPIs impeding antigen penetration of the oesophageal mucosa through epithelial repair and steroids blunting the allergy-based anti-inflammatory response. There are also data that PPIs inhibit different cytokines in EoE and GORD when compared with steroids, thus potentially and synergistically enhancing an antiinflammatory response. ${ }^{59}$

\section{Author affiliations}

1Department of Gastroenterology, Hospital San Pedro de Alcantara, Caceres, Spain ${ }^{2}$ Department of Gastroenterology and Hepatology, Academic Medical Centre, Amsterdam, The Netherlands

${ }^{3}$ Department of Pediatrics and Internal Medicine, Children's Health Children's Medical Center, and the University of Texas Southwestern Medical Center, Dallas, Texas, USA

${ }^{4}$ Center for Esophageal Diseases and Swallowing, University of North Carolina School of Medicine, Chapel Hill, North Carolina, USA

${ }^{5}$ Section of Pediatric Gastroenterology, Hepatology and Nutrition, Digestive Health Institute, Children's Hospital Colorado, Aurora, USA

${ }^{6}$ Section of Pediatric Gastroenterology, Hepatology and Nutrition, Indiana University School of Medicine, Riley Hospital for Children, Indianapolis, Indiana, USA

${ }^{7}$ Division of Gastroenterology and Hepatology, Department of Medicine, Northwestern University Feinberg School of Medicine, Chicago, Illinois, USA ${ }^{8}$ Division of Gastroenterology and Hepatology, Mayo Clinic, Rochester, Minnesota, USA

${ }^{9}$ Gastroenterology Service, Department of Medicine, Walter Reed National Military Medical Center, Bethesda, Maryland, USA

${ }^{10}$ Division of Allergy and Immunology, Cincinnati Children's Hospital Medical Center, Cincinnati, Ohio, USA

${ }^{11}$ Division of Gastroenterology and Hepatology, Centre Hospitalier Universitaire Vaudois, Lausanne, Switzerland

${ }^{12}$ Department of Internal Medicine, VA North Texas Health Care System, and the University of Texas Southwestern Medical Center, Dallas, Texas, USA

${ }^{13}$ Swiss EoE Research Network, Olten, Switzerland

${ }^{14}$ Department of Gastroenterology, Hospital General de Tomelloso, Tomelloso, Spain

Contributors All authors have equally contributed to drafting of the manuscript and critical revision of the manuscript for important intellectual content.

Competing interests AJB has received research funding by AstraZeneca, MMS, Endostim, Nutricia and Shire and received fees for development of educational material and speaker fees from MMS, AstraZeneca, Nutricia, Takeda and Astellas. EC and SIS: The Office of Medical Research, Departments of Veterans Affairs (SJS), the National Institutes of Health (R01-DK63621 to SJS, R01-CA134571 to SJS and K08-DK099383 to EC) and NASPGHAN Foundation/AstraZeneca Award (EC). ESD is consultant for Aptalis, Banner, Novartis, Receptos, Regeneron, Roche. Research funding from Meritage; Miraca; Receptos; Regeneron. Funding- NIH R01 DK101856. SKG is a consultant for Receptos and QOL. IH is a consultant for Shire, Regeneron, Roche and Receptos. He receives grant support from NIH U54 Al117804 (part of the Rare Disease Clinical Research Network (RDCRN), an initiative of the Office of Rare Disease Research (ORDR), NCATS and is funded through collaboration between NCATS, NIAID and NIDDK). JM-I is a consultant for Casen-Recordati. MER is a consultant for Novartis, Genetech, Receptos, Immune Pharmaceuticals, Celsus Therapeutics and NKT Therapeutics and has an equity interest in the latter three. He is an inventor of EoE-related patents owned by Cincinnati Children's Hospital Medical Center. He has a royalty interest in reslizumab, a drug being developed by Teva Pharmaceuticals. MER's component to this review was supported in part by NIH R37 Al045898, U19 Al070235, R01 Al057803, U54 Al117804, the Campaign Urging Research for Eosinophilic Disease (CURED), the Buckeye Foundation and 
Sunshine Charitable Foundation and its supporters, Denise A and David G Bunning. The U54 Al117804 is part of the Rare Disease Clinical Research Network (RDCRN), an initiative of the Office of Rare Disease Research (ORDR), NCATS and is funded through collaboration between NCATS, NIAID and NIDDK, which have collectively resulted in the Consortium of Eosinophilic Gastrointestinal Disease Researchers (CEGIR). ASc and ASt: Swiss National Science Foundation (32003B_160115/1). ASt has consultant contracts with Actelion, Falk, Novartis, Receptos, Regeneron and Roche-Genentech

Provenance and peer review Not commissioned; externally peer reviewed.

\section{REFERENCES}

1 Kandulski A, Malfertheiner P. Gastroesophageal reflux disease-from reflux episodes to mucosal inflammation. Nat Rev Gastroenterol Hepatol 2011;9:15-22.

2 Liacouras CA, Furuta GT, Hirano I, et al. Eosinophilic esophagitis: updated consensus recommendations for children and adults. J Allergy Clin Immunol 2011;128:3-20.

3 Kia L, Hirano I. Distinguishing GERD from eosinophilic esophagitis: concepts and controversies. Nat Rev Gastroenterol Hepatol 2015;12:379-86.

4 Molina-Infante J, Ferrando-Lamana L, Ripoll C, et al. Esophageal eosinophilic infiltration responds to proton pump inhibition in most adults. Clin Gastroenterol Hepatol 2011;9:110-17.

5 Straumann A, Aceves SS, Blanchard C, et al. Pediatric and adult eosinophilic esophagitis: similarities and differences. Allergy 2012;67:477-90.

6 Furuta GT, Liacouras CA, Collins MH, et al. Eosinophilic esophagitis in children and adults: a systematic review and consensus recommendations for diagnosis and treatment. Gastroenterology 2007;133:1342-63.

7 Dellon ES, Speck O, Woodward K, et al. Clinical and endoscopic characteristics do not reliably differentiate PPI-responsive esophageal eosinophilia and eosinophilic esophagitis in patients undergoing upper endoscopy: a prospective cohort study. Am J Gastroenterol 2013;108:1854-60.

8 Moawad FJ, Schoepfer AM, Safroneeva E, et al. Eosinophilic oesophagitis and proton pump inhibitor-responsive oesophageal eosinophilia have similar clinical, endoscopic and histological findings. Aliment Pharmacol Ther 2014;39:603-8.

9 Molina-Infante J, Rivas MD, Hernandez-Alonso $M$, et al. Remission in proton pump inhibitor-responsive esophageal eosinophilia correlates with down regulation of eotaxin-3 and TH2 cytokines, similarly to eosinophilic esophagitis after steroids. Aliment Pharmacol Ther 2014;40:955-65.

10 Iwakura N, Fujiwara Y, Tanaka F, et al. Basophil infiltration in eosinophilic oesophagitis and proton pump inhibitor-responsive oesophageal eosinophilia. Aliment Pharmacol Ther 2015;41:776-84.

11 Warners MJ, van Rhijn BD, Curvers WL, et al. PPI-responsive esophageal eosinophilia cannot be distinguished from eosinophilic esophagitis by endoscopic signs. Eur J Gastroenterol Hepatol 2015;27:506-11.

12 Hirano I, Moy N, Heckman MG, et al. Endoscopic assessment of the oesophageal features of eosinophilic oesophagitis: validation of a novel classification and grading system. Gut 2013;62:489-95.

13 Collins MH. Histopathologic features of eosinophilic esophagitis and eosinophilic gastrointestinal diseases. Gastroenterol Clin North Am 2014;43:257-68.

14 Dellon ES, Chen X, Miller CR, et al. Diagnostic utility of major basic protein, eotaxin-3, and leukotriene enzyme staining in eosinophilic esophagitis. Am J Gastroenterol 2012;107:1503-11.

15 Dellon ES, Speck O, Woodward K, et al. Markers of eosinophilic inflammation for diagnosis of eosinophilic esophagitis and proton pump inhibitor-responsive esophageal eosinophilia: a prospective study. Clin Gastroenterol Hepatol 2014;12:2015-22.

16 Sayej WN, Patel R, Baker RD, et al. Treatment with high-dose proton pump inhibitors helps distinguish eosinophilic esophagitis from noneosinophilic esophagitis. J Pediatr Gastroenterol Nutr 2009;49:1-7.

17 Isomoto H, Nishi Y, Wang A, et al. Mucosal concentrations of proinflammatory cytokines and chemokines at gastric cardia: implication of Helicobacter pylori infection and gastroesophageal reflux. Am J Gastroenterol 2004;99:1063-8.

18 Rothenberg ME. Molecular, genetic, and cellular bases for treating eosinophilic esophagitis. Gastroenterology 2015;148:1143-57.

19 Wen T, Stucke EM, Grotjan TM, et al. Molecular diagnosis of eosinophilic esophagitis by gene expression profiling. Gastroenterology 2013;145:1289-99.

20 Moawad FJ, Wells JM, Johnson RL, et al. Comparison of eotaxin-3 biomarker in patients with eosinophilic oesophagitis, proton pump inhibitor-responsive oesophageal eosinophilia and gastro-oesophageal reflux disease. Aliment Pharmacol Ther 2015;42:231-8.

21 van Rhijn BD, Weijenborg PW, Verheij J, et al. Proton pump inhibitors partially restore mucosal integrity in patients with proton pump inhibitor-responsive esophageal eosinophilia but not eosinophilic esophagitis. Clin Gastroenterol Hepatol 2014;12:1815-23.

22 Wen T, Dellon ES, Moawad FJ, et al. Transcriptome analysis of proton pump inhibitor-responsive esophageal eosinophilia reveals proton pump inhibitor-reversible allergic inflammation. J Allergy Clin Immunol 2015;135:187-97.
23 Butz BK, Wen T, Gleich GJ, et al. Efficacy, dose reduction, and resistance to high-dose fluticasone in patients with eosinophilic esophagitis. Gastroenterology 2014;147:324-33.e5.

24 Rothenberg ME, Spergel JM, Sherrill JD, et al. Common variants at 5 q22 associate with pediatric eosinophilic esophagitis. Nat Genet 2010;42:289-91.

25 Kottyan LC, Davis BP, Sherrill JD, et al. Genome-wide association analysis of eosinophilic esophagitis provides insight into the tissue specificity of this allergic disease. Nat Genet 2014;46:895-900.

26 Sleiman PMA, Wang ML, Cianferoni A, et al. GWAS identifies four novel eosinophilic esophagitis loci. Nat Commun 2014;5:5593.

27 Ngo P, Furuta GT, Antonioli DA, et al. Eosinophils in the esophagus-peptic or allergic eosinophilic esophagitis? Case series of three patients with esophageal eosinophilia. Am J Gastroenterol 2006;101:1666-70.

28 Desai TK, Stecevic V, Chang $\mathrm{CH}$, et al. Association of eosinophilic inflammation with esophageal food impaction in adults. Gastrointest Endosc 2005;61:795-801.

29 Dranove JE, Horn DS, Davis MA, et al. Predictors of response to proton pump inhibitor therapy among children with significant esophageal eosinophilia. J Pediatr 2009;154:96-100.

30 Peterson KA, Thomas KL, Hilden K, et al. Comparison of esomeprazole to aerosolized, swallowed fluticasone for eosinophilic esophagitis. Dig Dis Sci 2010;55:1313-19.

31 Moawad FJ, Veerappan GR, Dias JA, et al. Randomized controlled trial comparing aerosolized swallowed fluticasone to esomeprazole for esophageal eosinophilia. Am J Gastroenterol 2013;108:366-72.

32 Vazquez-Elizondo G, Ngamruengphong S, Khrisna M, et al. The outcome of patients with oesophageal eosinophilic infiltration after an eight-week trial of a proton pump inhibitor. Aliment Pharmacol Ther 2013;38:1312-19.

33 Gutiérrez-Junquera C, Fernández-Fernández S, Cilleruelo ML, et al. High prevalence of response to proton pump inhibitor treatment in children with esophageal eosinophilia. J Pediatr Gastroenterol Nutr Published Online First 23 Oct 2015. doi: 10.1097/MPG.0000000000001019

34 Lucendo AJ, Arias A, Molina-Infante J. Efficacy of proton pump inhibitor drugs for inducing clinical and histological remission in patients with symptomatic esophageal eosinophilia: a systematic review and meta-analysis. Clin Gastroenterol Hepatol Published Online First: 3 Aug 2015. doi: 10.1016/j.cgh.2015.07.041

35 Dohil R, Newbury RO, Aceves S. Transient PPI responsive esophageal eosinophilia may be a clinical sub-phenotype of pediatric eosinophilic esophagitis. Dig Dis Sci 2012:57:1413-19.

36 Schroeder S, Capocelli KE, Masterson JC, et al. Effect of proton pump inhibitor on esophageal eosinophilia. J Pediatr Gastroenterol Nutr 2013;56:166-72.

37 Molina-Infante J, Rodriguez-Sanchez J, Martinek J, et al. Long-term loss of response in proton pump inhibitor-responsive esophageal eosinophilia is uncommon and influenced by CYP2C19 genotype and rhinoconjunctivitis. Am I Gastroenterol Published Online First: 29 Sep 2015. doi: 10.1038/ajg.2015.314

38 Cheng E, Souza RF, Spechler SJ. Eosinophilic esophagitis: interactions with gastroesophageal reflux disease. Gastroenterol Clin North Am 2014;43:243-56.

39 Kedika RR, Souza RF, Spechler SJ. Potential anti-inflammatory effects of proton pump inhibitors: a review and discussion of the clinical implications. Dig Dis Sci 2009:54:2312-17.

40 Cheng E, Zhang $X$, Huo $X$, et al. Omeprazole blocks eotaxin-3 expression by oesophageal squamous cells from patients with eosinophilic oesophagitis and GORD. Gut 2013;62:824-32

41 Zhang $X$, Cheng E, Huo $X$, et al. Omeprazole blocks STAT6 binding to the eotaxin-3 promoter in eosinophilic esophagitis cells. PLoS One 2012;7:e50037.

42 Park JY, Zhang $X$, Nguyen $N$, et al. Proton pump inhibitors decrease eotaxin-3 expression in the proximal esophagus of children with esophageal eosinophilia. PLoS One 2014;9:e101391.

43 Sherrill JD, KC K, Wu D, et al. Desmoglein-1 regulates esophageal epithelial barrier function and immune responses in eosinophilic esophagitis. Mucosal Immunol 2014;7:718-29.

44 van Rhijn BD, Verheij J, van den Bergh Weerman MA, et al. Histological response to fluticasone propionate in patients with eosinophilic esophagitis is associated with improved functional esophageal mucosal integrity. Am I Gastroenterol 2015;110:1289-97.

45 Spechler SJ, Genta RM, Souza RF. Thoughts on the complex relationship between gastroesophageal reflux disease and eosinophilic esophagitis. Am I Gastroenterol 2007;102:1301-6.

46 Krarup AL, Villadsen GE, Mejlgaard E, et al. Acid hypersensitivity in patients with eosinophilic oesophagitis. Scand J Gastroenterol 2010;45:273-81.

47 Francis DL, Foxx-Orenstein A, Arora AS, et al. Results of ambulatory pH monitoring do not reliably predict response to therapy in patients with eosinophilic esophagitis. Aliment Pharmacol Ther 2012;35:300-7.

48 Abe $Y$, lijima $K$, Ohara $S$, et al. A Japanese case series of 12 patients with oesophageal eosinophilia. J Gastroenterol 2011;46:25-30.

49 Levine J, Lai J, Edelman $M$, et al. Conservative long-term treatment of children with eosinophilic esophagitis. Ann Allergy Asthma Immunol 2012;108:363-6.

50 Sodikoff J, Hirano I. Proton pump inhibitor-responsive esophageal eosinophilia does not preclude food-responsive eosinophilic esophagitis. J Allergy Clin Immunol Published Online First: 25 Aug 2015. doi: 10.1016/j.jaci.2015.07.008 
51 Lucendo AJ, Arias A, Gonzalez-Cervera J, et al. Dual response to dietary/topical steroid and PPI therapy in adult patients with eosinophilic esophagitis. J Allergy Clin Immunol 2015. doi: 10.1016/j.jaci.2015.07.033

52 Katzka D. Eosinophilic esophagitis and proton pump-responsive esophageal eosinophilia: what is in a name? Clin Gastroenterol Hepatol 2014;12:2023-5.

53 Dellon ES, Gonsalves N, Hirano I, et al. ACG Clinical Guideline: evidence based approach to the diagnosis and management of esophageal eosinophilia and eosinophilic esophagitis. Am J Gastroenterol 2013;108:679-92.

54 Dellon ES, Liacouras CA. Advances in clinical management of eosinophilic esophagitis. Gastroenterology 2014;147:1238-54.

55 Chuang MY, Chinnaratha MA, Hancock DG, et al. Topical steroid therapy for the treatment of eosinophilic esophagitis (EoE): a systematic review and meta-analysis. Clin Trans/ Gastroenterol 2015;6:e82.
56 Arias A, Gonzalez-Cervera J, Tenias JM, et al. Efficacy of dietary interventions for inducing histologic remission in patients with eosinophilic esophagitis: a systematic review and meta-analysis. Gastroenterology 2014; 146:1639-48.

57 Calabrese C, Bortolotti M, Fabbri A, et al. Reversibility of GERD ultrastructural alterations and relief of symptoms after omeprazole treatment. Am J Gastroenterol 2005; 100:537-42.

58 Straumann A, Schoepfer A. Update on basic and clinical aspects of eosinophilic oesophagitis. Gut 2014;63:1355-63.

59 Zhang $X$, Huo X, Yu C, et al. Omeprazole and fluticasone inhibit IL-13-stimulated eotaxin-3 expression by esohageal epithelial cells through different mechanisms and with additive effects: rationale for combining PPIs with topical steroids for EoE patients. Gastroenterology 2015;148(Suppl 1):S-51. 